\title{
THREE-DIMENSIONAL GRAPHICAL EXERGY ANALYSIS OF A DISTILLATION COLUMN
}

\author{
ARIEF BUDIMAN AND MASARU ISHIDA \\ Research Laboratory of Resources Utilization, \\ Tokyo Institute of Technology, Yokohama 226
}

Key Words: Exergy Analysis, Exergy Loss, Three-Dimensional Graphics, Distillation, Integrated EUD

\begin{abstract}
This paper demonstrates a three-dimensional graphical representation of exergy losses in a distillation column by applying an Energy-Utilization Diagram (EUD). The concepts of both premixing model and individual energy level are applied to classify the exergy loss in a column into six kinds; losses due to heating and mixing in liquid phase, losses due to cooling and mixing in vapor phase, and losses due to evaporation of light components and condensation of heavy components through phase changes. The diagram displays transformed energy, its energy level, and unit height of the column in three-dimensional space. This diagram is useful for qualifying and quantifying the exergy loss in a distillation column, and the target to minimize the energy and exergy consumption can be identified. The characteristics of close-to-equilibrium points (CEP) are also demonstrated.
\end{abstract}

\section{Introduction}

Distillation is a separation process that consumes a large amount of energy. Hence, energy saving becomes the prime task for process designers. Thermodynamic or exergy analyses of a distillation column have been done, but most of them were concerned with binary mixtures where the equilibrium concentration is uniquely related to the bubble-point temperature of the mixture. Recently, the effectiveness of the exergy concept was discussed (Ognisty, T.P., 1995). One of the authors, Ishida, and Taprap (1992) have introduced the premixing concept to disclose individual processes in a complex phenomenon and distinguished the overall process into individual subprocesses in vapor and liquid phases and in phase changes as well. Furthermore, in order to clarify the phase change of each component in the mixture, the individual energy level concept was proposed (Ishida and Taprap, 1993). Then, an Energy-Utilization Diagram (EUD) in which the energy level and the amount of transformed energy of both the energy donor and the energy acceptor are drawn on a two-dimensional domain was applied to represent the information on exergy for each plate of a distillation column graphically. This EUD has explained nicely the features of exergy losses on each plate in a distillation column in detail. However, it cannot give integrated information for the whole column.

This paper is to develop three-dimensional graphic exergy analysis by representing the exergy loss as a volume in three dimensions instead of area on two

* Received on February 15, 1996. Correspondence concerning this article should be addressed to M. Ishida. dimensions. This methodology reveals the transferred energy and the accompanied exergy loss of each subprocess on each plate, as well as the distribution of those values over the whole column. Locations of close-to-equilibrium points (CEP) are also demonstrated.

\section{Model Calculation}

\subsection{Simulation of a non-equilibrium column}

The classical procedure to design a distillation column was to assume the equilibrium state on each plate. In an actual column, however, such ideal behavior cannot be achieved. Hence, to predict actual behavior and column performance, a nonequilibrium (sometimes called mass transfer) model

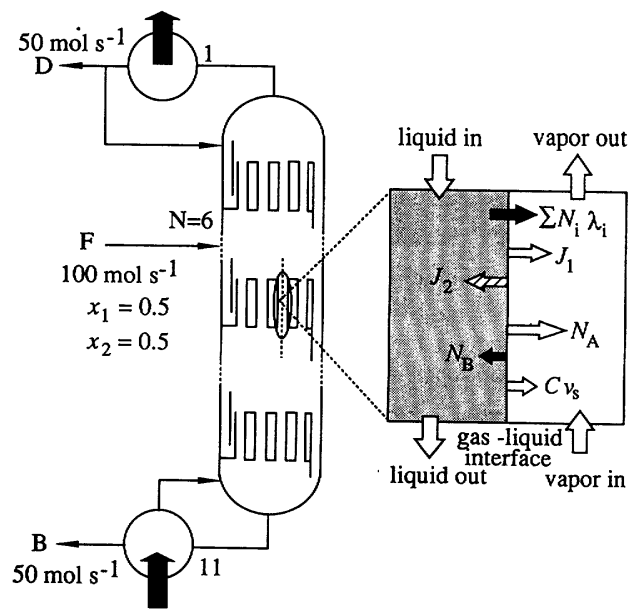

Fig. 1 Physical picture of mass transfer in a distillation column 


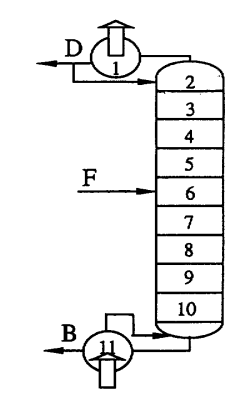

(a) Schematic illustration of column

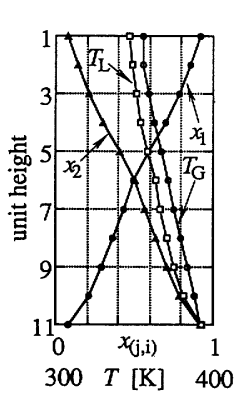

(b) Profiles of liquid mole fraction and temperature

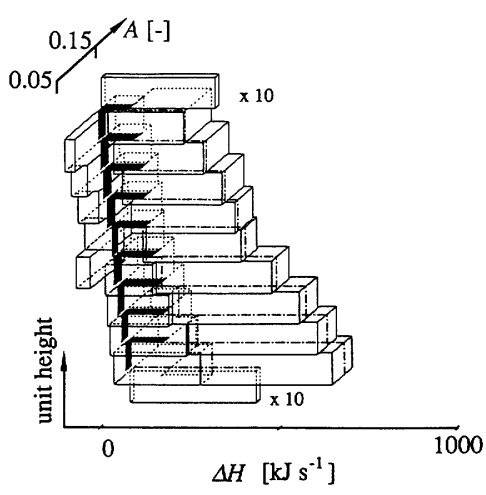

(c) Integrated Energy-Utilization Diagram

Fig. 2 Qualitative approach to an Integrated EUD for $\mathrm{nC}_{6} \mathrm{H}_{14^{-}}$ $\mathrm{nC}_{8} \mathrm{H}_{18}$ mixture $(R=1.5)$

was proposed by Ito and Asano (1982). The key feature of the model is that mass and heat transfer, especially in the gas phase, control the rates, so mass and heat balances together with mass flux and equilibrium relations for the interface are taken into consideration, as illustrated in Fig. 1. The actual mass flux in the gas film, $N_{i}$ for component $i$, is evaluated as the sum of the diffusional flux, $J_{i}$, and the flux caused by convective flow, $y_{i} \cdot C \cdot v_{\mathrm{s}}$, at the interface between liquid and vapor.

A distillation column with 11 plates is considered for simplicity in this study. A binary mixture with $n$-hexane as the light component (component 1) and $n$-octane as the heavy component (component 2) was chosen. The condenser (plate 1) and the reboiler (plate 11) are treated as an equilibrium stage. Mixtures with equal mole fractions for both components $\left(x_{1}=x_{2}=0.5\right)$ were fed at the middle column (plate 6) at the rate of $100 \mathrm{~mol} \mathrm{~s}^{-1}$. The flowrate of the distillate was set at $50 \mathrm{~mol} \mathrm{~s}^{-1}$ and the pressure of the column was assumed to be $1 \mathrm{~atm}$. We assume that the area of the column is $1.0 \mathrm{~m}^{2}$, the height of the liquid phase is $0.1 \mathrm{~m}$, and that each plate is a perforated tray with 10,000 holes of $3 \mathrm{~mm}$ diameter.

\subsection{Integrated energy-utilization diagram (Integrated EUD)}

Figure 2(a) represents a schematic demonstration of the present distillation column, (b) illustrates its associated concentration profiles in terms of liquid mole fractions of $n$-hexane and $n$-octane and its temperature profile for the base case of the reflux ratio $R=1.5$, and (c) exhibits the Integrated EUD proposed in this study. The arrangement of each plate described in Fig. 2(a) is related to the unit height of (b) and (c).

This Integrated EUD is composed of many rectangular blocks, and the volume of each block indicates the amount of exergy loss of each subprocess. Hence the total volume of the blocks represents the total exergy loss in a distillation column. The blocks in the same level represent the exergy loss of the plate corresponding to the plate number shown in Fig. 2(a). For the top block (plate 1: condenser) and the bottom block (plate 11: reboiler), the scale of the abscissa is reduced to $1 / 10$. The meaning of each block will be demonstrated in detail in the following section. The advantage of this three-dimensional display of the exergy loss distribution in a distillation column is that this display may be used even when the number of plates is increased. This is true especially when six kinds of blocks are represented by different colors and various angles on a CRT screen.

\section{Characteristics of Integrated EUD}

\subsection{EUD for a single plate}

As shown in a previous paper (Ishida and Taprap, 1992), the exergy loss on a plate in a distillation column may be classified into six subprocesses as shown in Table 1. The exergy losses caused by mixing in the vapor phase as well as in the liquid phase are calculated based on the premixing concept, while the exergy losses caused by evaporation of each light component as well as by condensation of each heavy component are obtained based on the concept of individual energy level (Ishida and Taprap, 1993).

When these concepts are applied to plate 8, for example, the exergy loss on this plate is represented as shown in Fig. 3(a). This diagram is called an EnergyUtilization Diagram (abbreviated as EUD) (Ishida and Taprap, 1993). The abscissa of this diagram represents the amount of energy released or accepted, while the ordinate $A$ indicates the energy level of this released or accepted energy. Let us examine the rectangle denoted as $L_{\mathrm{c}}$. The value $\mathrm{d}$ represents the energy level of the cooling of the gas phase introduced 
Table 1 Exergy loss profile for the base condition $(R=1.5)$

\begin{tabular}{|c|c|c|c|c|c|c|c|}
\hline \multirow{2}{*}{ Plate } & \multirow{2}{*}{$Q$} & \multicolumn{2}{|c|}{ EXL in liq. phase } & \multicolumn{2}{|c|}{ EXL vap. phase } & \multicolumn{2}{|c|}{ EXL in phase change } \\
\hline & & Heating & Mixing & Cooling & Mixing & Comp. 1 & Comp. 2 \\
\hline 1 & 3898.48 & - & - & 42.8839 & - & - & - \\
\hline 2 & & 0.0627 & 2.6736 & 0.3000 & 1.9066 & 0.7922 & 11.3453 \\
\hline 3 & & 0.1223 & 3.1189 & 0.3736 & 2.3395 & 1.2551 & 12.0506 \\
\hline 4 & & 0.2227 & 3.6684 & 0.4201 & 2.7167 & 1.8405 & 11.9318 \\
\hline 5 & & 0.3732 & 4.1068 & 0.4115 & 2.8580 & 2.3757 & 10.4599 \\
\hline \multirow[t]{2}{*}{6} & & 0.0960 & 3.4771 & 0.3610 & 2.7631 & 2.7363 & 8.5258 \\
\hline & & $0.1466 *$ & $0.0342 *$ & - & - & - & - \\
\hline 7 & & 0.4318 & 2.7392 & 0.4502 & 3.9341 & 4.3889 & 10.6630 \\
\hline 8 & & 0.7133 & 3.9858 & 0.4977 & 5.2425 & 6.6178 & 11.9535 \\
\hline 9 & & 1.0323 & 5.1275 & 0.4691 & 6.2606 & 9.0635 & 11.5897 \\
\hline 10 & & 1.3007 & 6.1273 & 0.3879 & 7.0651 & 11.1633 & 9.6167 \\
\hline 11 & 4175.97 & 47.1832 & - & - & - & - & - \\
\hline
\end{tabular}

All value are in $\mathrm{kJ} / \mathrm{s}$, and ${ }^{*}$ denotes exergy loss for feed stream

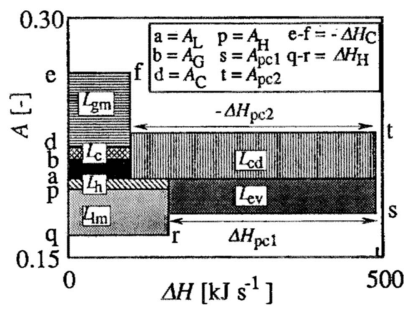

(a) $\triangle H-A$ diagram

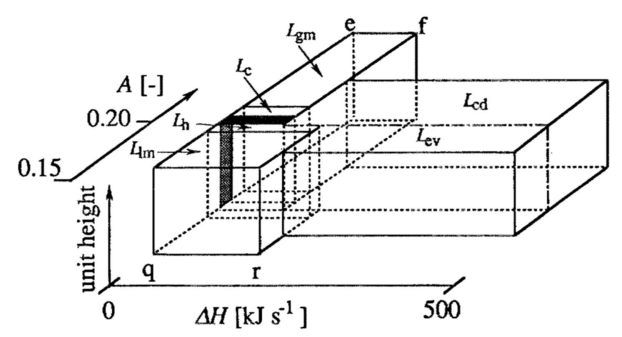

(b) $\Delta H$-A-unit height diagram

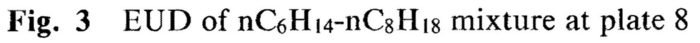

from the lower plate 9, while $\mathrm{b}$ is the energy level $A_{\mathrm{G}}$ of the gas phase temperature $T_{\mathrm{G}}$ of the present plate 8 . The width e-f represents the amount of heat released by this cooling. Hence the height of rectangle $L_{\mathrm{c}}$ represents the average drop in the energy level of the sensible heat of the gas phase, and the area of this rectangle is equal to the exergy loss caused by this cooling.

The rectangle $L_{\mathrm{gm}}$ indicates the exergy loss of mixing in the vapor phase. For ideal gas, the heat of mixing is zero. Hence, this rectangle only has meaning in its area and neither its width nor its ordinate have any meaning.

Similarly, the rectangle $L_{\mathrm{h}}$ denotes the exergy loss of heating of the liquid stream. The value $p$ represent the energy level of the heating of the liquid phase introduced from the upper plate 7 , while a is the energy level $A_{\mathrm{L}}$ of the liquid phase temperature $T_{\mathrm{L}}$ of the present plate 8 . Its width $\mathrm{q}-\mathrm{r}$ indicates the amount of heat accepted for this heating. Hence the height of the rectangle $L_{\mathrm{h}}$ represents the average rise in the energy level of the sensible heat of the liquid phase. The rectangle $L_{\mathrm{lm}}$ only has meaning in its area and it shows the exergy loss of mixing of the liquid phase. Generally, there is some gap between the gas and liquid temperatures for each plate, and it indicates the extent of deviation from equilibrium. This gap is denoted by a black belt or a black rectangle.

The rectangle $L_{\mathrm{cd}}$ is related to condensation of heavy components. Its width $-\Delta H_{\mathrm{pc} 2}$ denotes the amount of heat released by this condensation. Its energy level $t$ is obtained by the individual energy level concept proposed in a previous paper (Ishida and Taprap, 1993). Since the released heat is accepted as heat at the liquid temperature $T_{\mathrm{L}}$, the area of this rectangle represents the exergy loss caused by this condensation.

On the contrary, the rectangle $L_{\mathrm{ev}}$ is related to evaporation of the light component. Its width $\Delta H_{\mathrm{pcl}}$ indicates the amount of heat accepted by this evaporation. Its energy level sis obtained by the individual energy level in the manner similar to level $t$ for condensation. This heat is supplied from the liquid at its temperature $T_{\mathrm{L}}$, and hence the area of this rectangle becomes equal to the exergy loss caused by this evaporation.

Since the heat loss from the column wall is assumed to be zero in this study, the total amount of both the heat released and the heat accepted for plate 8 is equal to $500 \mathrm{~kJ} \mathrm{~s}^{-1}$.

Then, this two-dimensional presentation of the characteristic features of the quantity and the quality of various kinds of energy in Fig. 3(a) may be changed to a three-dimensional presentation as shown in Fig. 


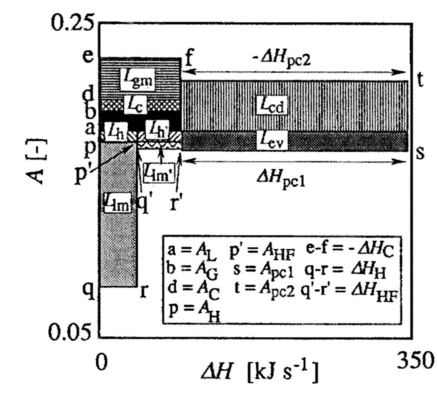

(a) $\triangle H-A$ diagram

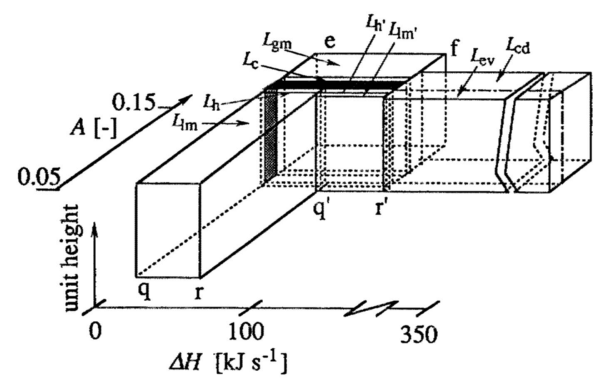

(b) $\Delta H-A$-unit height diagram

Fig. 4 EUD of $\mathrm{nC}_{6} \mathrm{H}_{14}-\mathrm{nC}_{8} \mathrm{H}_{18}$ mixture at plate 6 (feed plate)

3(b). The $\Delta H$-axis and $A$-axis, respectively, in (b) correspond to the abscissa and the ordinate in (a). All rectangular blocks have the same height on $z$-axis, i.e., the unit height, so that the exergy loss given as the area in Fig. 3(a) is represented as a volume in (b). The driving force for each subprocess, which is represented as the gap between the level of donated energy and that of the accepted energy, is, however, maintained as the gap on the $y$-axis of $A$ in this threedimensional presentation.

Figure 4(a) and (b) shows the characteristics of the energy transformation at the feed plate. The feed liquid of the specified composition $\left(x_{1}=x_{2}=0.5\right.$ in this example) is assumed to be introduced at the temperature of its bubble point and is mixed with the liquid on this plate. This means that two streams of quite different compositions are mixed with the liquid on this plate. Hence, a large exergy loss is generally accompanied in the liquid phase on this plate.

In this particular case, the temperature of the feed stream $(356.5 \mathrm{~K})$ is lower than that of the feed plate liquid $(358.7 \mathrm{~K})$. So this feed stream receives heat and hence its rectangle, block $L_{\mathrm{h}^{\prime}}$, appears below level a (i.e., the level of the liquid temperature $T_{\mathrm{L}}$ on this plate). There may be a case in which the temperature of the feed stream is higher than $T_{\mathrm{L}}$ of the feed plate. Then, this feed stream is cooled and its rectangle appears above level a. The exergy loss of mixing in this feed stream is denoted by rectangle $L_{\mathrm{lm}}$.

\subsection{EUD for a whole column}

The mole fraction of the heavy component, $n$ -

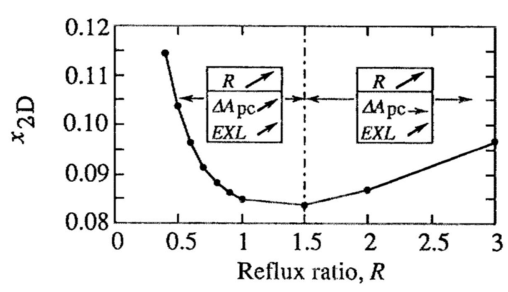

(a) Separation at different reflux ratio

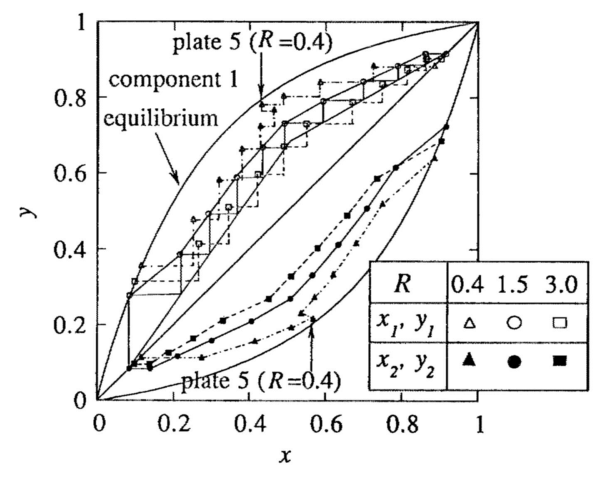

(b) $x$-y diagram at different reflux ratio

Fig. 5 Performance of separation at different reflux ratios

octane, in the distillate, $x_{2, \mathrm{D}}$, changes with the reflux ratio $R$, as shown in Fig. 5(a) and the corresponding $x$ $y$ diagrams at three typical reflux ratios are shown in (b). The latter figure also displays the equilibrium lines $\left(y_{i, \text { eq }}=K_{i} x_{i}\right)$ for $n$-hexane (as component 1$)$ and $n$-octane (as component 2).

Table 1 summarizes the exergy losses of six kinds of subprocesses on each plate for $R=1.5$, i.e., exergy losses due to heating and mixing in the liquid phase, those due to cooling and mixing in the vapor phase, and those due to phase changes, i.e., evaporation of light component 1 and condensation of heavy component 2. Figure 5(a) shows that the minimum value of $x_{2, \mathrm{D}}$ is obtained around $R=1.5$ and we may say that this base case, $R=1.5$, gives the best separation performance. $Q$ in Table 1 denotes the amount of heat removed from condenser (plate 1) or heat added to the reboiler (plate 11). For the feed plate (plate 6), the exergy losses caused by the feed stream are presented separately. All the tabulated values are calculated by the mass transfer model except the condenser and the reboiler, for which equilibrium is assumed.

The exergy losses represented as volume in Fig. 2(c) and their values in Table 1 indicate the following tendencies for the whole column:

Liquid phase The exergy loss of heating, which appears at the front of $A_{\mathrm{L}}$ in Fig. 2(c), is generally quite small. The amount of heat required for this heating, width q-r in Fig. 3, increases sharply with the plate number, because the liquid flow rate at the lower plates (e.g., $159.7 \mathrm{~mol} \mathrm{~s}^{-1}$ at plate 10$)$ is much higher 
than that at the upper plates $\left(e . g ., 73.2 \mathrm{~mol} \mathrm{~s}^{-1}\right.$ at plate 2).

The exergy loss of mixing in the liquid phase, which appears also at the front of $A_{\mathrm{L}}$ in Fig. 2(c), increases slightly with the plate number at the rectifying section, but sharply increases at the stripping section. This is also caused by large liquid flow rates at the lower plates.

Vapor phase The exergy loss of cooling, which appears at the rear of $A_{\mathrm{L}}$ in Fig. 2(c), is generally not significant. The amount of heat released by cooling of vapor, width e-f in Fig. 3, increases only slightly with the plate number. This change can be explained by the fact that the vapor flow rate at plate 10 is $109.7 \mathrm{~mol} \mathrm{~s}^{-1}$ and that at plate 2 is $123.2 \mathrm{~mol} \mathrm{~s}^{-1}$.

The exergy loss of mixing in the vapor phase, which also appears at the rear of $A_{\mathrm{L}}$ in Fig. 2(c), has the same tendency as the exergy loss of mixing in the liquid phase

Phase change The exergy loss of evaporation of light component, $n$-hexane, appears at the front of $A_{\mathrm{L}}$, while that of condensation of heavy component, $n$ octane, appears at the rear of $A_{\mathrm{L}}$. They increase at both rectifying and stripping sections with the plate number at this base case, $R=1.5$.

In the rectifying section, $\Delta H_{\mathrm{pc} l}$ is larger than $-\Delta H_{\mathrm{pc} 2}$, it means that the heat required by evaporation of the light component exceeds that released by condensation of the heavy component. On the other hand, in the stripping section, $-\Delta H_{\mathrm{pc} 2}$ is larger than $\Delta H_{\mathrm{pcl}}$, indicating that the heat released by condensation of the heavy component exceeds the heat required by evaporation of the light component. The difference between $A_{\mathrm{L}}$ and $A_{\mathrm{pcl}},\left(A_{\mathrm{L}}-A_{\mathrm{pcl}}\right)$, may be considered as the driving force of evaporation of the light component. It is the smallest at plate 2 , and it increases with plate number. Conversely, the driving force of condensation of the heavy component, $\left(A_{\mathrm{pc} 2}-\right.$ $A_{\mathrm{L}}$ ), decreases with the plate number. It is found that the driving force of evaporation of the light component, say at plate 2 , is much smaller than that of the heavy component, say at plate 10 . In this base case of a binary system, the concentration of component 1 in the distillate $\left(x_{1}=0.916\right)$ is exactly the same as to that of component 2 in the bottoms $\left(x_{2}=0.916\right)$, while the condition of component 1 at plate 2 is much closer to the equilibrium line than that of component 2 at plate 10. Closeness to equilibrium gives a smaller driving force.

Finally, the total exergy loss on one plate can be represented by adding all these six kinds of blocks. This sum on each plate increases gradually from plate 2 to plate 10, as seen in Fig. 2(c) or Table 1. The exergy loss of cooling in the condenser is calculated by assuming a heat sink at the bubble-point temperature of the distillate. When the average temperature of this heat sink becomes lower than this temperature, the exergy loss in the condenser will be increased further. On the other hand, the exergy loss of heating in the reboiler is calculated by assuming a heat source at the dew-point temperature of the bottoms. When the average temperature of this heat source becomes higher than this temperature, the exergy loss in the reboiler will be increased, too. As seen in Table 1 , the exergy losses at the reboiler and the condenser are very large. In Fig. 2(c), these losses are shown by the scale of $1 / 10$ because of their high values.

It is to be noted that the total exergy loss in this base case, $306.8 \mathrm{~kJ} \mathrm{~s}^{-1}$, becomes equal to that derived by the following equation (Ishida, 1995):

$$
\begin{aligned}
E X L & =T_{\mathrm{o}} \sum_{\mathrm{j}} \Delta S_{\mathrm{j}} \\
& =T_{\mathrm{o}}\left(\Delta S_{\text {process }}+\Delta S_{\text {heat source }}+\Delta S_{\text {heat sink }}\right)
\end{aligned}
$$

Here $\Delta S_{\text {process }}$ means the entropy change of separation per unit time given by the value of ( $S$ of distillate $+S$ of bottoms $-S$ of feed), and $\Delta S_{\text {heat source }}$ and $\Delta S_{\text {heat sink, }}$ respectively, are given by $-Q / T$ and $Q / T$.

\subsection{Effect of the reflux ratio}

Decreasing the reflux ratio As described previously, the reflux ratio $R=1.5$ gives the minimum concentration of component 2 in the distillate. Then, by reducing this reflux ratio, we reach $R=0.4$ as the minimum reflux ratio. The profile of the liquid mole fraction and the temperature at this condition can be seen in Fig. 6(a), and the exergy loss distribution on an Integrated EUD in (b). The corresponding relations between $x$ and $y$ on each plate are shown in Fig. 5(b).

Comparison of Figs. 6(b) and 2(c) explains that the exergy losses on each plate are much decreased by reduction of the reflux ratio, especially on the middle plates. Hence, Fig. 6(b) gives the distribution with relatively large exergy losses at the top and bottom plates and with quite small exergy losses at the middle plates. Figure 5(b) shows that the points $(x, y)$ on these middle plates are close to equilibrium curve. Especially, plate 5 gives a point nearly on the equilibrium line. Hence, we may say that plate 5 is the close-to-equilibrium point (CEP) and this point determines the minimum reflux ratio. Since the concentration of the liquid on plate 5 which flows into the feed plate becomes close to that of feed, the exergy loss of mixing in the liquid phase on plate 6 is decreased prominently.

Let us look at plate 5 , the close-to-equilibrium point (CEP), on Fig. 6(b). Not only the exergy loss, but also the driving force for the evaporation of light component 1 is very small. This CEP generally appears at the plate (plate 5) above the feed plate (plate 6). Also, the temperature of gas $T_{\mathrm{G}}$ approaches that of liquid $T_{\mathrm{L}}$ at plate 5 , and hence the gap between $A_{\mathrm{G}}$ and $A_{\mathrm{L}}$ represented by a black belt becomes negligibly small.

By looking at Figs. 2(c) and 6(b) focusing on the 


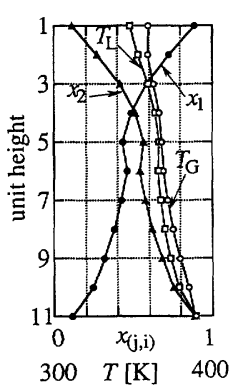

(a) Profiles of liquid mole fraction and temperature

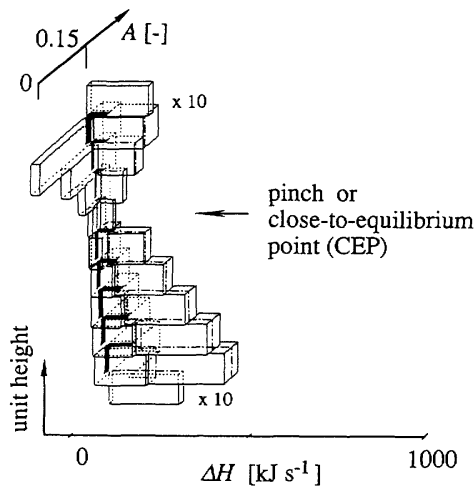

(b) Integrated Energy-Utilization Diagram

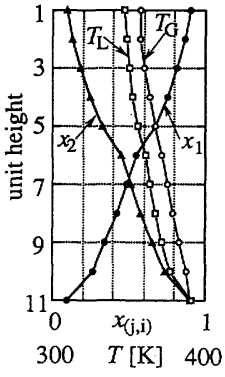

(a) Profiles of liquid mole fraction and temperature

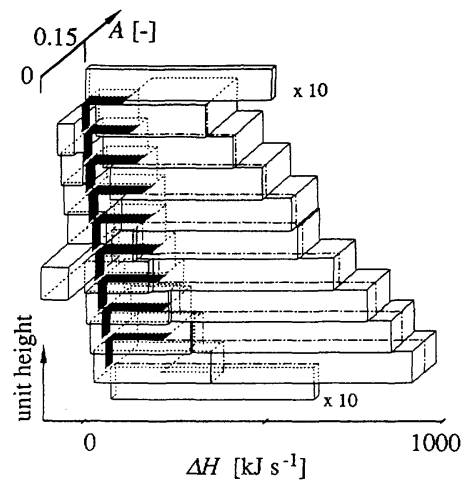

(b) Integrated Energy-Utilization Diagram

Fig. 6 Qualitative approach to an Integrated EUD for $\mathrm{nC}_{6} \mathrm{H}_{14}-\mathrm{nC}_{8} \mathrm{H}_{18}$ mixture $(R=0.4)$

Fig. 7 Qualitative approach to an Integrated EUD for $\mathrm{nC}_{6} \mathrm{H}_{14}-\mathrm{nC}_{8} \mathrm{H}_{18}$ mixture $(R=3)$

exergy loss and the driving force for evaporation of light component 1 on each plate, we can find out that they both increase with the increase in the reflux ratio. Hence we may say that the increase in the reflux ratio gives the increase in the exergy loss, but that this increase in exergy loss plays a role of increasing the driving force of evaporation, resulting in good separation performance (i.e., low concentration of component 2 in the distillate) as illustrated in Fig. 5(a) when the reflux ratio is less than 1.5.

Also, we may say from Figs. 2(c), 6(b) and 5(a) that the small driving force of evaporation at the middle plates has a more prominent effect on the separation performance in Fig. 5(a) than that at plate 2. We have $x_{1}=0.725$ at plate 2 and $x_{1}=0.432$ at plate 5. The smaller values of the mole fraction of light component 1 may have given this tendency.

Increasing the reflux ratio The profiles of the liquid mole fraction and the temperature, and the exergy loss distribution on an Integrated EUD at condition $R=3$ are seen in Fig. 7(a) and (b), respectively. Comparison of Figs. 7 and 2 shows that the exergy losses in the liquid phase (heating and mixing), in the gas phase (cooling and mixing), and in the phase change (evaporation and condensation) are increased by the increase in the reflux ratio. But it is to be noted that the driving force for evaporation of light component 1 remains nearly constant. Hence we may say that the increase in the reflux ratio above $R=1.5$ gives the increase in the exergy losses, but that this increase in the exergy losses cannot increase the driving force of evaporation, resulting in poor separation performance (i.e., high concentration of component 2 in the distillate) as seen in Fig. 5(a).

In the equilibrium calculation, the minimum reflux ratio gives the lowest separation performance, while the total reflux gives the best one. For the mass transfer model, however, too much of an increase in

the reflux ratio makes the gas phase velocity through holes with the fixed hole area so high that the contact time of the gas phase with the liquid phase on each plate becomes very short. This condition corresponds to the situation that an increase in exergy losses cannot contribute to an increase in the driving force of evaporation. Hence, the optimal reflux ratio is observed.

We have mainly demonstrated the exergy losses of subprocesses and the role of the driving force of evaporation of the light component. However, the present three-dimensional visualization may have wide applications, for example, in elucidating the effect of side heating or side cooling.

\section{Conclusion}

1. Three-dimensional graphic exergy analysis based on Integrated EUD can display characteristic features of exergy over the whole distillation column.

2. The distribution of both the exergy losses and the driving forces of six subprocesses can be seen compactly and clearly.

3. By reducing the reflux ratio, the middle-neck distribution can be seen. This neck plate is the closeto-equilibrium point (CEP).

4. The optimal reflux ratio is observed in the simulation. In the region less than the optimal ratio, the increase in the reflux ratio is accompanied by the increase in the total exergy loss. But this exergy loss increases the driving force of evaporation. In the region more than the optimal ratio, the increase in the reflux ratio is only accompanied by the increase in the total exergy loss, while the driving force of evaporation remains nearly constant.

$\begin{array}{llr}\text { Nomenclature } & \\ A & = & \text { energy level } \\ C & = & {[-]}\end{array}$




$\begin{array}{ll}E X L & =\text { Exergy loss } \\ H & =\text { enthalpy } \\ Q & =\text { heat added at reboiler or removed from } \\ & =\text { condenser } \\ S & =\text { temperature } \\ T & =\text { velocity of convective flow } \\ v_{\mathrm{s}} & =\text { mole fraction in liquid phase } \\ x & =\text { mole fraction in vapor phase } \\ y & =\text { cooling } \\ <\mathrm{subscript} & > \\ \mathrm{c} & =\text { condensation } \\ \mathrm{cd} & =\text { equilibrium } \\ \mathrm{eq} & =\text { gas/vapor phase } \\ \mathrm{ev} & =\text { mixing in gas phase } \\ \mathrm{G} & =\text { heating } \\ \mathrm{gm} & =\text { heating at feed stream } \\ \mathrm{h} & =i \text {-th component } \\ \mathrm{hf} & \end{array}$

$\begin{array}{lll}{\left[\mathrm{J} \mathrm{s}^{-1}\right]} & \mathrm{L} & =\text { liquid phase } \\ {\left[\mathrm{J} \mathrm{s}^{-1}\right]} & \mathrm{lm} & =\text { mixing in liquid phase } \\ & \mathrm{pc} & =\text { phase change }\end{array}$

Literature Cited

Ishida, M.; Thermodynamics-Its perfect comprehension and applications - (in Japanese), Baihukan, Tokyo, Japan (1995)

Ishida, M. and R. Taprap; "Application of Energy Utilization Diagram for Graphic Exergy Analysis of Multicomponent Distillation Columns," J. Chem. Eng. Japan, 25, 396-402 (1992)

Ishida, M. and R. Taprap; "Introduction of Individual Energy Level for Exergy Analysis of Process System with Multiple Component," J. Chem. Eng. Japan, 26, 437-439 (1993)

Ito, A. and K. Asano; "Thermal Effect in Non-Adiabatic Binary Distillation;" Effect of Partial Condensation of Mixed Vapor on the Rates of Heat and Mass Transfer and Prediction of H.T.U," Chem . Eng. Sci., 37, 1007-1014 (1982)

Ognisty, T.P.; "Analyze Distillation Column with Thermodynamics," Chem. Eng. Progress, 91, 40-46 (1995) 